\title{
E-cadherin expression in a rat model of acute pancreatitis
}

\author{
WEIHONG YUAN ${ }^{1 *}$, QI PAN ${ }^{1 *}$, GUOCHANG CHEN $^{1}$, JUN YAN $^{2}$, JIAZENG XIA $^{3}$ and YIGANG CHEN ${ }^{3}$ \\ ${ }^{1}$ Department of Gastroenterology, Yixing People's Hospital, Yixing, Jiangsu 214200; ${ }^{2}$ Department of General Surgery, \\ Shanghai Jiao Tong University Affiliated Sixth People's Hospital, Shanghai 200233; ${ }^{3}$ Department of General Surgery, \\ Nanjing Medical University Affiliated Wuxi Second Hospital, Wuxi, Jiangsu 214002, P.R. China
}

Received August 29, 2014; Accepted July 14, 2015

DOI: $10.3892 /$ etm.2015.2786

\begin{abstract}
A clinical requirement exists for early biomarkers that can predict the severity of acute pancreatitis (AP). In order to determine whether E-cadherin is associated with the severity of AP, a pancreatitic rat model was established and the expression levels of E-cadherin were detected. A study population of 24 Sprague Dawley rats was administered intraperitoneal injections of various concentrations of L-arginine in order to induce pancreatitis. Rats were assigned to the severe acute pancreatitis (SAP) or mild acute pancreatitis (MAP) group based on the results of histological evaluations and the serum levels of amylase. An additional 8 rats received intraperitoneal injections of $\mathrm{NaCl}$ solution, as a control group. For each group, the serum concentrations of soluble E-cadherin and the expression levels of E-cadherin protein in the pancreatic tissue were detected. The results indicated that the rat model of pancreatitis was successfully established. Rats in the high concentration L-arginine treatment group, which exhibited a higher pancreatitis pathology score and level of serum amylase, were assigned to the SAP group. Low concentration L-arginine group rats were assigned to the MAP group. The pathology scores and levels of serum amylase in the SAP and MAP group rats were higher compared with the control group rats. The levels of serum E-cadherin were the most elevated in the SAP group. Statistically significant differences were detected in the SAP and MAP groups compared with the control group, and in the SAP group compared with the MAP group $(\mathrm{P}<0.05)$. Furthermore, the levels of E-cadherin protein in the pancreatic tissue were elevated in the SAP group compared with the MAP and control groups. In conclusion, the present study demonstrated that E-cadherin was overex-
\end{abstract}

Correspondence to: Professor Yigang Chen or Dr Jiazeng Xia, Department of General Surgery, Nanjing Medical University Affiliated Wuxi Second Hospital, 68 Zhongshan Road, Wuxi, Jiangsu 214002, P.R. China

E-mail: iamyigang@126.com

E-mail:xjz_wuxi@alumni.sjtu.edu.cn

${ }^{*}$ Contributed equally

Key words: pancreatitis, severe acute pancreatitis, mild acute pancreatitis, E-cadherin pressed in SAP rats, and the overexpression of E-cadherin may be associated with the severity of AP.

\section{Introduction}

Acute pancreatitis (AP) is a severe inflammatory disease that accounts for $>220,000$ hospital admissions in the United States each year (1). Although there has been recent progress in the diagnosis and treatment of AP, the incidence of AP has not decreased and its serious systemic complications are a worldwide health issue (2). In certain cases, severe AP (SAP) may lead to multiple organ failure, and thus the mortality rate among patients with SAP may approach 30\% (3). Therefore, it is crucial to identify effective early diagnostic markers for AP, and particularly for SAP. The level of serum amylase is a commonly-used clinical indicator for the severity of AP; however, this level not particularly sensitive and it is often difficult to obtain a pancreatic specimen from patients with AP for pathological analysis $(4,5)$.

E-cadherin is a calcium-dependent cell-cell adhesion molecule that has been linked to cancer development and inflammatory disorders $(6,7)$. During the inflammatory process, cell-cell contacts tend to dissolve, which permits the unregulated movement of fluids and electrolytes into the interstitial space, resulting in tissue edema (8). Once AP has been initiated, the appearance of inflammatory infiltration and interstitial edema are common features (9). Therefore, it is hypothesized that the expression levels of E-cadherin fluctuate during the progression of AP. The aim of the present study was to establish an animal model of pancreatitis and evaluate the levels of soluble E-cadherin, in order to assess its efficacy as a marker of AP severity.

\section{Materials and methods}

Pancreatitic animal model and experimental design. Animal experiments were authorized according to the Guidance Suggestions for the Care and Use of Laboratory Animals issued by the Ministry of Science and Technology of the People's Republic of China, and were approved by the Ethics Committee of the Nanjing Medical University Affiliated Wuxi Second Hospital (Wuxi, China). Sprague Dawley (SD) rats were supplied by the Sino-British SIPPR/BK Laboratory Animal Co., Ltd., (Shanghai, China). Animal experiments were conducted as described in previous studies $(10,11)$ at 
Nanjing Medical University Affiliated Wuxi No. 2 People's Hospital between June 2013 and July 2014. In brief, 24 healthy adult male Sprague Dawley (SD) rats, weighing $170 \pm 15.5 \mathrm{~g}$, were randomly assigned into two treatment groups $(n=12$ per group). In the high concentration L-arginine (HCLa) group, each SD rat received two intraperitoneal injections of $20 \%$ L-arginine (Sinopharm Chemical Reagent Co., Ltd., Shanghai, China; $2.5 \mathrm{~g} / \mathrm{kg}$ body weight), at an interval of $1 \mathrm{~h}$. In the low concentration L-arginine (LCLa) group, each SD rat received two intraperitoneal injections of $6 \% \mathrm{~L}$-arginine $(2.5 \mathrm{~g} / \mathrm{kg}$ body weight), at an interval of $1 \mathrm{~h}$. At $24 \mathrm{~h}$ after the induction of pancreatitis using L-arginine, all SD rats were sacrificed under ether anesthesia. The pancreas of each rat was then rapidly removed, and separated from the surrounding lymph nodes and fat. Each pancreatic tissue sample was divided into two parts: One part was fixed in $10 \%$ buffered formaldehyde for histological evaluation and the second part was reserved for protein extraction. The severity of the pancreatitis was determined based on the histological scoring, including the degrees of edema, inflammation, vacuolization and necrosis, and by measurement of serum amylase levels. Furthermore, 8 additional rats received intraperitoneal injections of an equal quantity of $0.9 \% \mathrm{NaCl}$ solution, and were designated as the control group.

Histological evaluation. Pancreatic samples were fixed in $10 \%$ buffered formaldehyde, cut into $5 \mu \mathrm{m}$ sections, and stained with hematoxylin and eosin (H\&E; Maixin Biotech Co., Ltd., Fuzhou, China) and assessed blindly by two senior pathologists (Qi Pan and Guochang Chen). The tissue sections were graded as described previously (12). A total of 10 high-power fields (HPF) of each section were selected at random and were visualized under a CX31 microscope (Olympus America Inc., Center Valley, PA, USA). Samples were scored on a scale of $0-4$, according to the degree of edema, necrosis, vacuolization and inflammation. The degree of edema was scored as follows: 0 , No edema; 1 , diffuse expansion of interlobar septa; 2 , severe diffuse expansion of interlobar septa; 3 , diffuse expansion of interacinar septa; and 4, diffuse expansion of intercellular septa. The degree of inflammation was scored as follows: 0, No inflammation; 1, inflammation around ductal margin; 2 , inflammation in parenchyma ( $<50 \%$ of lobules); 3 , inflammation in parenchyma (50-75\% of lobules); and 4 , inflammation in parenchyma ( $>75 \%$ of lobules). The degree of vacuolization was scored as follows: 0, No vacuolization; 1, peroductal vacuolization $(<5 \%)$; 2 , focal vacuolization $(5-20 \%)$; 3 , diffused vacuolization (21-50\%); and 4, severe vacuolization $(>50 \%)$. The degree of necrosis was scored as follows: 0 , No necrosis; 1, 1-4 necrotic cells/HPF; 2, 5-10 necrotic cells/HPF; 3, 11-15 necrotic cells/HPF; and 4, >16 necrotic cells/HPF. The final score was calculated as the sum of the results of all parameters (including degrees of edema, necrosis, vascularization and inflammation). The final score was between 0 and 16 .

Serum levels of amylase. During the animal experiments, a 2-ml blood sample was extracted from each rat following anesthetization. The blood samples were centrifuged at 1,509 x g for $15 \mathrm{~min}$, and stored at $-80^{\circ} \mathrm{C}$ until further use. The levels of serum amylase were measured using a Hitachi 7600-020 automatic biochemical analyzer (Hitachi, Ltd., Tokyo, Japan) (13).
Serum levels of E-cadherin. A 2-ml blood sample was extracted from each rat following anesthetization. The levels of E-cadherin were measured using a commercially available MK117 E-cadherin ELISA kit (Takara Bio, Inc., Otsu, Japan).

Western blot analysis to determine the expression of E-cadherin protein. Whole cell lysates were prepared from pancreatic tissue specimens. Briefly, pancreatic tissue samples were homogenized using a glass-on-glass tissue homogenizer. Subsequently, the homogenates were centrifuged $\left(10 \mathrm{~min}, 4^{\circ} \mathrm{C}\right.$, $11,750 \mathrm{x} \mathrm{g}$ ) and the supernatants were collected. Total protein was extracted using a Qproteome Mammalian Protein Prep kit (Qiagen GmbH, Hilden, Germany). The levels of E-cadherin protein in the pancreatic tissue were measured using Coomassie blue staining (Maixin Biotech Co., Ltd.). A standard western blot assay was performed using a primary goat polyclonal antibody against rat E-cadherin (dilution, 1:1,000; catalog no. ab1416; Abcam, Cambridge, UK) and an anti-goat IgG antibody, which was a horseradish peroxidase-linked $\mathrm{F}(\mathrm{ab})_{2}$ fragment obtained from a rabbit (dilution 1:2,000; catalog no. BA1060; Wuhan Boster Biological Technology, Ltd., Wuhan, China). The loading of equal quantities of protein samples was verified using an anti- $\beta$-actin antibody (dilution, 1:1,000; catalog no. sc-47778; Santa Cruz Biotechnology, Inc., Dallas, TX, USA), with a secondary antibody (dilution, 1:2,000; cat. no. BA1051; Wuhan Boster Biological Technology, Ltd.), as a loading control. The proteins under investigation were detected using the Pierce Enhanced Chemiluminescence Western Blotting substrate (Thermo Fisher Scientific, Inc., Waltham, MA, USA). The intensity of the bands was visualized using an Amersham enhanced chemiluminescence system (ImageQuant LAS 4000; GE Healthcare Bio-Sciences, Pittsburgh, PA, USA), according to the manufacturer's instructions.

Statistical analysis. Statistical analysis was performed using SPSS software, version 18.0 (SPSS Inc., Chicago, IL, USA). The experimental values were analyzed using the paired-samples $t$-test and were expressed as the mean \pm standard deviation. One-way analysis of variance (ANOVA) was calculated to determine the differences between groups for each parameter at each time point. Non-parametrical Kruskal-Wallis tests were used when equal variances were not assumed in one-way ANOVA. $\mathrm{P}<0.05$ was considered to indicate a statistically significant difference.

\section{Results}

Histological analysis. No rats died unexpectedly following the intraperitoneal injections, and the rats were sacrificed at $24 \mathrm{~h}$ after the injections. In the control group ( $\mathrm{NaCl}$-treated), no pancreatic edema or peritoneal exudate were detected (Fig. 1A). $\mathrm{H} \& \mathrm{E}$ staining of the control group pancreatic tissue revealed no significant swelling, inflammation or necrosis (Fig. 2A). In the LCLa group, edema of the pancreas and necrosis of the fat surrounding the pancreas were observed in all rat tissue samples (Fig. 1B). H\&E staining revealed interlobular edema and inflammatory cell infiltration in the pancreatic tissue (Fig. 2B). In the HCLa group, edema, hemorrhage, necrosis of the pancreas and bloody ascites were observed in all rat tissue 
Table I. Histological scoring for rats with acute pancreatitis.

\begin{tabular}{lccccc}
\hline Group & Edema & Inflammation & Vacuolization & Necrosis & Score \\
\hline Control & $0.65 \pm 0.22$ & $0.44 \pm 0.19$ & $0.35 \pm 0.06$ & $0.31 \pm 0.09$ & $1.75 \pm 0.16$ \\
LCLa & $3.52 \pm 2.13$ & $2.34 \pm 1.65$ & $0.45 \pm 0.07$ & $0.44 \pm 0.13$ & $6.75 \pm 2.12^{\mathrm{a}}$ \\
HCLa & $3.08 \pm 1.77$ & $3.24 \pm 1.65$ & $1.85 \pm 0.92$ & $2.21 \pm 0.88$ & $10.38 \pm 0.56^{\mathrm{a}}$ \\
\hline
\end{tabular}

Data are present as the mean \pm standard deviation. ${ }^{a} \mathrm{P}<0.05$ vs. control group. LCLa, low concentration L-arginine group; HCLa, high concentration L-arginine group.

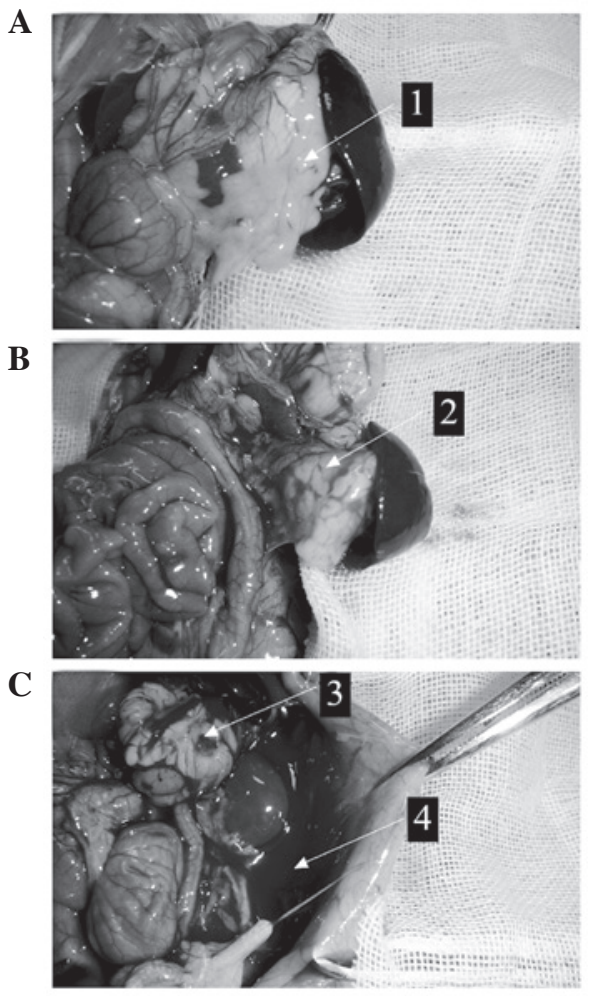

Figure 1. Differences in the pancreatic tissues between the (A) control, (B) low concentration L-arginine (LCLa) and (C) high concentration L-arginine (HCLa) groups. Arrow 1 indicates normal pancreas tissue in the control group. Arrow 2 indicates edema of the pancreas and necrosis of fat around the pancreas tissue in the LCLa group. Arrow 3 indicates edema, hemorrhage and necrosis of a pancreas tissue in the HCLa group, and arrow 4 indicates bloody ascites in the HCLa group.

samples (Fig. 1C). Furthermore, the destruction of pancreatic lobules, interstitial edema, vascular congestion and infiltration of neutrophils and monocytes were observed in the HCLa group samples (Fig. 2C).

Pathology scores for the HCLa $(10.38 \pm 0.56)$ and LCLa $(6.75 \pm 2.12)$ groups were higher compared with the control group (1.75 \pm 0.16$)$. Statistically significant differences were detected in the HCLa and LCLa groups compared with the control group, and in the HCLa group compared with the LCLa group $(\mathrm{P}<0.05)$. These results are presented in Table I and Fig. 3.

Serum levels of amylase. The levels of serum amylase were higher in the HCLa $(1,497.29 \pm 319.57 \mathrm{U} / 1)$ and LCLa $(1,003.83 \pm 111.52 \mathrm{U} / \mathrm{l})$ groups compared with the control
A

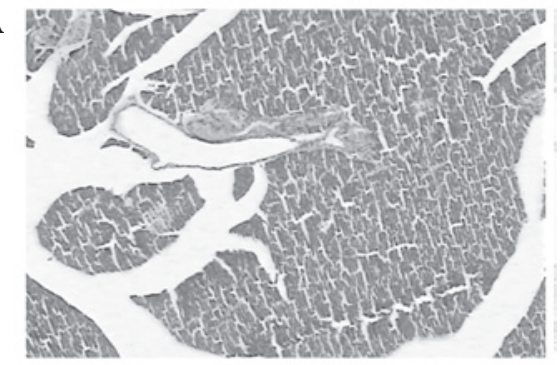

B

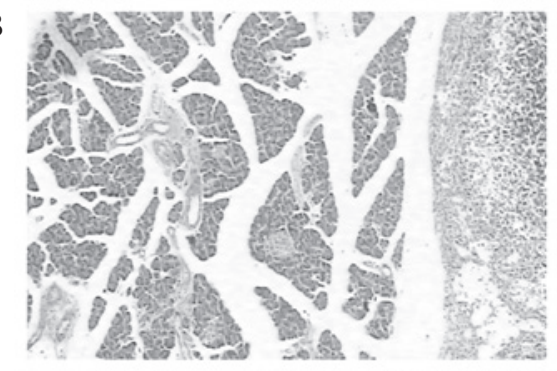

C

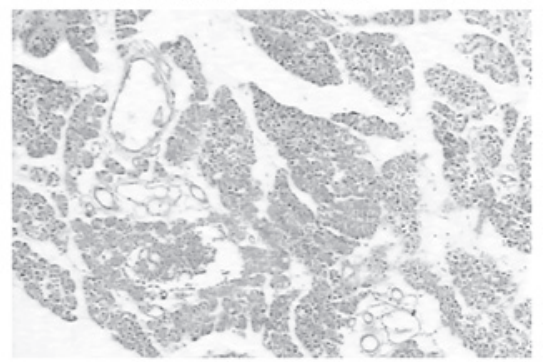

Figure 2. Hematoxylin and eosin-stained pancreatic tissue from Sprague Dawley rats in the (A) control, (B) low concentration L-arginine (LCLa) and (C) high concentration L-arginine (HCLa) groups. No significant pancreatic swelling, inflammation or necrosis was observed in the control group. Interlobular edema and inflammatory cell infiltration was observed in the pancreatic tissue of the LCLa group. Destruction of the pancreatic lobules, interstitial edema, vascular congestion and inflammatory cell infiltration were observed in the HCLa group.

group (301.75 $\pm 26.89 \mathrm{U} / 1)$. Statistically significant increases in serum amylase were detected in the HCLa and LCLa groups compared with the control group, and in the HCLa group compared with the LCLa group $(\mathrm{P}<0.05)$. The results are presented in Fig. 4.

SAP and mild AP (MAP) groups. All the HCLa and LCLa group rats exhibited inflammation and edema of the pancreas. However, hemorrhage and bloody ascites were only observed in the HCLa group rats. The most elevated pancreatitis pathology scores and serum amylase levels were observed in the HCLa group rats. In addition, the pathology scores and 


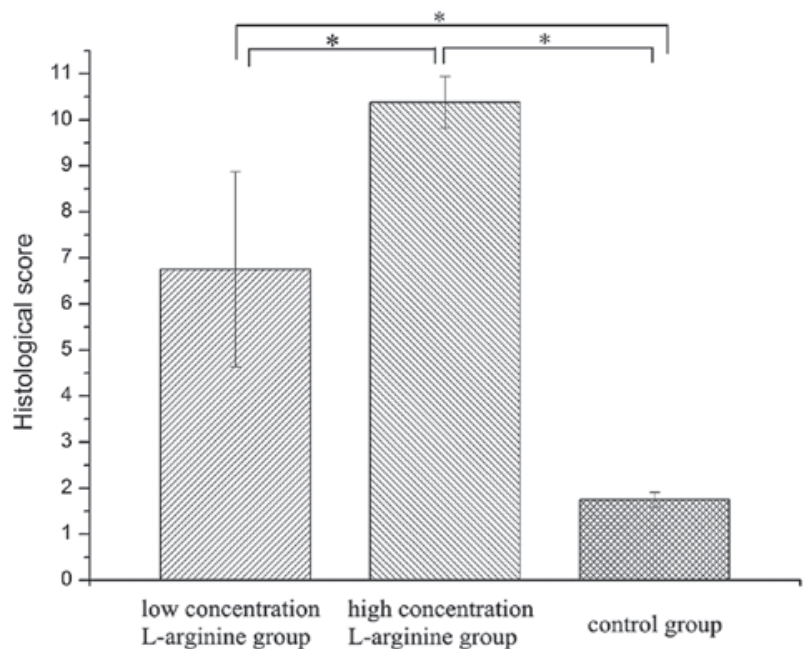

Figure 3. Pathology scores for the low concentration L-arginine, high concentration L-arginine and control groups. ${ }^{*} \mathrm{P}<0.05$.

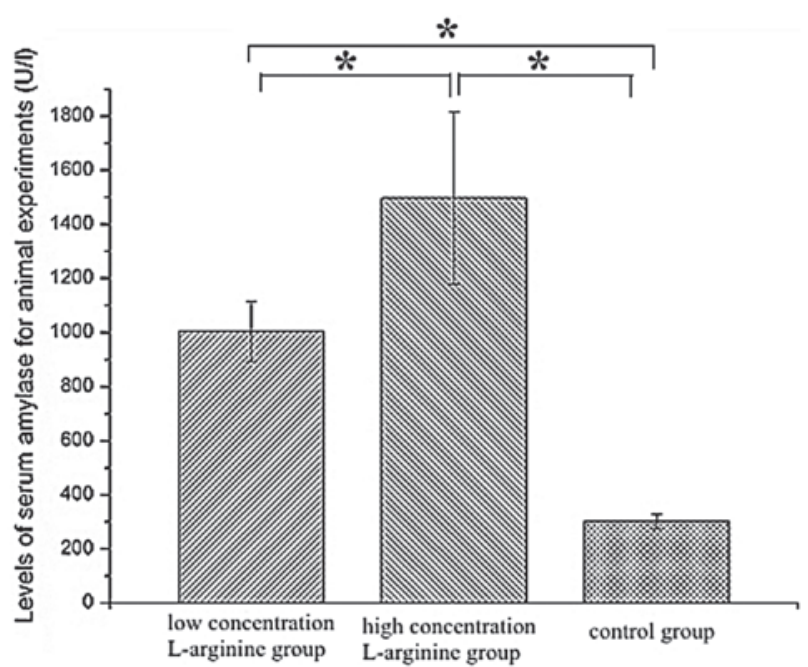

Figure 4. Levels of serum amylase for the low concentration L-arginine, high concentration L-arginine and control groups. ${ }^{*} \mathrm{P}<0.05$.

levels of serum amylase in the HCLa and LCLa group rats were higher compared with the control group rats. Therefore, the HCLa rats were assigned as the SAP group, and the LCLa rats were assigned as the MAP group.

Serum levels of E-cadherin. The levels of E-cadherin were highest in the SAP group rats. In addition, the levels of E-cadherin were higher in the $\operatorname{SAP}(1,561.75 \pm 144.82 \mathrm{pg} / \mathrm{ml})$ and MAP $(1,025.50 \pm 131.33 \mathrm{pg} / \mathrm{ml})$ groups compared with the control group $(626.50 \pm 72.12 \mathrm{pg} / \mathrm{ml} ; \mathrm{P}<0.05)$. Statistically significant elevations in serum E-cadherin were detected in the MAP and SAP groups compared with the control group $(\mathrm{P}<0.05)$, and in the SAP group compared with the MAP group $(\mathrm{P}<0.05)$. The results are presented in Fig. 5.

Expression of E-cadherin protein. The expression of E-cadherin protein was evaluated using western blot analysis. The levels of E-cadherin protein were higher in the SAP group compared with the MAP and control groups

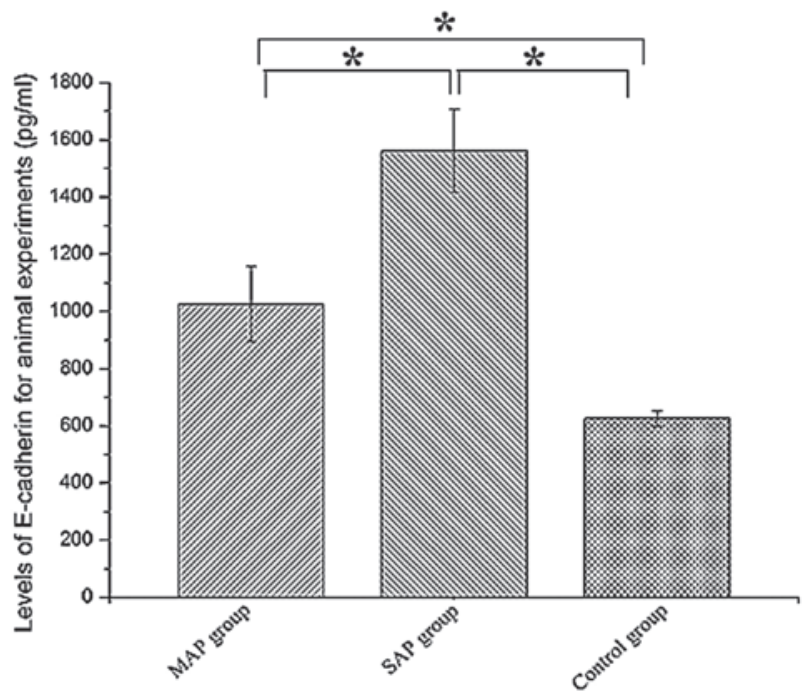

Figure 5. Levels of E-cadherin in the MAP, SAP and control groups. " $\mathrm{P}<0.05$. MAP, mild acute pancreatitis; SAP, severe acute pancreatitis.

A
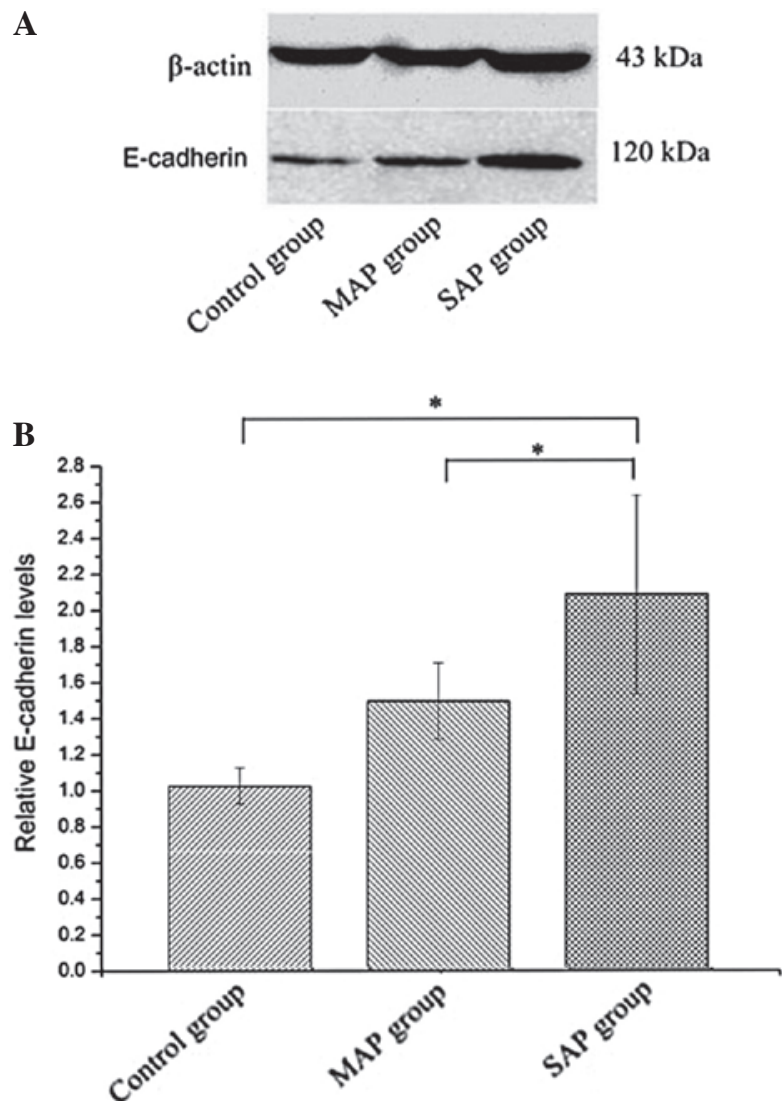

Figure 6. Western blot analysis results for E-cadherin protein expression levels in the control, MAP and SAP groups. (A) Differential expression of E-cadherin protein in the control, MAP and SAP groups. (B) Statistical results of the levels of E-cadherin protein in the control, MAP and SAP groups. ${ }^{*} \mathrm{P}<0.05$. MAP, mild acute pancreatitis; SAP, severe acute pancreatitis.

(Fig. 6). Statistically significant differences in E-cadherin protein expression were detected between the SAP and MAP group rats, and between the SAP and control group rats. No statistically significant differences in E-cadherin protein expression were detected between the MAP and control group rats. 


\section{Discussion}

To date, a number of inflammatory molecules, have been identified as potential predictive markers of SAP, including interleukin (IL)-1, IL-6 and IL-10 (14,15). However, the majority of studies on E-cadherin have focused on the development and recurrence of cancer (16), and thus, few studies have investigated the role played by E-cadherin in inflammation. To the best of our knowledge, the role of E-cadherin in pancreatitis remains controversial. In 2009, Sewpaul et al observed that the mean concentration of soluble E-cadherin in patients with SAP at $<12 \mathrm{~h}$ was significantly higher compared with that in healthy volunteers and patients with other gastrointestinal pathologies (17). However, Pezzilli et al reported that the serum levels of E-cadherin were not associated with the severity of AP (18).

In the present study, a rat model of pancreatitis was successful established using L-arginine. The SAP and MAP groups were designated according to the results of histological evaluations and the serum levels of amylase. The levels of serum E-cadherin and E-cadherin protein were highest in rats in the SAP group, which were evidently increased compared with the levels detected in rats in the MAP and control groups. These results suggest that the expression of E-cadherin is associated with the severity of AP.

The mechanism underlying E-cadherin overexpression in rats with SAP remains unclear. It is possible that E-cadherin-associated apoptosis may serve a crucial function in the pathogenesis of SAP. Takeyama reported that apoptosis was involved in the mechanism of infectious complications, in addition to organ dysfunction, in SAP (19). It is known that apoptosis is regulated by numerous genes, and E-cadherin is one among a variety factors that may influence the apoptotic process (20). During apoptosis, E-cadherin is efficiently cleaved in epithelial cells, and fragments with apparent molecular masses of 24, 29 and $84 \mathrm{kDa}$ are generated following the induction of apoptosis by staurosporine or camptothecin (21).

Another possible cause of the overexpression of E-cadherin is that it may contribute to the extravasation of erythrocytes and leukocytes in the early stages of SAP $(8,21)$. In the present rat experiments, hemorrhage of the pancreas and bloody ascites were observed in the SAP group rats. The process of extravasation into the parenchyma involves a number of molecular interactions between blood cells and endothelial cells, including tight adhesions, rolling and diapedesis (22). Vonlaufen et al reported that, in cerulein-induced AP, the junctional adhesion molecule $\mathrm{C}$ is upregulated (23). In addition, Mayerle et al demonstrated that a variant that was $15 \mathrm{kDa}$ smaller than E-cadherin, which was detected in the early stage of pancreatitis, was the product of E-cadherin cleavage at amino acid 394 in the extracellular domain; this phenomenon was consistent with E-cadherin cleavage by leukocyte-produced elastase (8). Therefore, previous results suggested that polymorphonuclear leukocyte-released elastase may be involved in the dissociation of cell-cell contacts, the extracellular cleavage of E-cadherin and, ultimately, the transmigration of leukocytes into the epithelial tissue during the initial phase of experimental pancreatitis (8).

In conclusion, the role of E-cadherin in pancreatitis remains unclear. However, the present results indicate that the expression levels of E-cadherin in SAP rats were higher compared with those in MAP rats. Therefore, E-cadherin may be associated with the severity of AP.

\section{Acknowledgements}

The present study was supported by grants from the National Natural Science Foundation of China (nos. 30901422 and 51271117).

\section{References}

1. Talukdar R and Vege SS: Recent developments in acute pancreatitis. Clin Gastroenterol Hepatol 7 (Suppl 11): 3-9, 2009.

2. Zeng YB, Zhan XB, Guo XR, et al: Risk factors for pancreatic infection in patients with severe acute pancreatitis: An analysis of 163 cases. J Dig Dis 15: 377-385, 2014.

3. Granger J and Remick D: Acute pancreatitis: models, markers and mediators. Shock 24 (Suppl 1): 45-51, 2005.

4. Pezzilli R, Billi P, Miglioli M and Gullo L: Serum amylase and lipase concentrations and lipase/amylase ratio in assessment of etiology and severity of acute pancreatitis. Dig Dis Sci 38: 1265-1269, 1993.

5. Pooran N, Indaram A, Singh P and Bank S: Cytokines (IL-6, IL-8, TNF): early and reliable predictors of severe acute pancreatitis. J Clin Gastroenterol 37: 263-266, 2003.

6. Saito T, Yoshida K, Matsumoto K, et al: Inflammatory cytokines induce a reduction in E-cadherin expression and morphological changes in MDCK cells. Res Vet Sci 96: 288-291, 2014.

7. Ahmed RA, Shawky Ael-A, and Hamed RH: Prognostic significance of cyclin D1 and E-cadherin expression in laryngeal squamous cell carcinoma. Pathol Oncol Res 20: 625-633, 2014.

8. Mayerle J, Schnekenburger J, Krüger B, et al: Extracellular cleavage of E-cadherin by leukocyte elastase during acute experimental pancreatitis in rats. Gastroenterology 129: 1251-1267, 2005.

9. Glasbrenner B and Adler G: Pathophysiology of acute pancreatitis. Hepatogastroenterology 40: 517-521, 1993.

10. Dawra R and Saluja AK: L-arginine-induced experimental acute pancreatitis. The Pancreapedia: Exocrine Pancreas Knowledge Base, 2012.

11. Czakó L, Takács T, Varga IS, et al: The pathogenesis of L-arginine induced acute necrotizing pancreatitis: inflammatory mediators and endogenous cholecystokinin. J Physiol Paris 94: 43-50, 2000.

12. Rongione AJ, Kusske A, Kwan K, Ashley SW, Reber HA and McFadden DW: Interleukin 10 reduces the severity of acute pancreatitis in rats. Gastroenterology 112: 960-967, 1997.

13. Yan S, Ding Y, Sun F, Lu Z, Xue L, Liu X, Shuai M, Fang C, Wang $\mathrm{Y}$, Cheng $\mathrm{H}$, et al: Pretreatment of cisplatin in recipients attenuates post-transplantation pancreatitis in murine model. Int J Biol Sci 8: 298-309, 2012.

14. Matull W, Pereira S and O'Donohue J: Biochemical markers of acute pancreatitis. J Clin Pathol 59: 340-344, 2006.

15. Mayer J, Rau B, Gansauge F and Beger HG: Inflammatory mediators in human acute pancreatitis: clinical and pathophysiological implications. Gut 47: 546-552, 2000.

16. Wijnhoven B, Dinjens W and Pignatelli M: E-cadherin-catenin cell-cell adhesion complex and human cancer. Br J Surg 87: 99-1005, 2000.

17. Sewpaul A, French J, Khoo T, Kernohan M, Kirby J and Charnley R: Soluble E-cadherin: an early marker of severity in acute pancreatitis. HPB Surgery 2009: 6, 2009.

18. Pezzilli R, Corsi MM, Barassi A, et al: Serum E-cadherin and hepatocyte growth factor in acute pancreatitis: Exploring time course and severity assessment. Immunogastroenterology 2: 57-61, 2013.

19. Takeyama Y: Significance of apoptotic cell death in systemic complications with severe acute pancreatitis. J Gastroenterol 40: $1-10,2005$.

20. Steinhusen U, Weiske J, Badock V, Tauber R, Bommert K and Huber O: Cleavage and shedding of E-cadherin after induction of apoptosis. J Biol Chem 276: 4972-4980, 2001.

21. Vestweber D: Regulation of endothelial cell contacts during leukocyte extravasation. Curr Opin Cell Biol 14: 587-593, 2002.

22. Imhof BA and Dunon D: Leukocyte migration and adhesion. Advances in immunology 58: 345, 1995.

23. Vonlaufen A, Aurrand-Lions M, Pastor CM, et al: The role of junctional adhesion molecule C (JAM-C) in acute pancreatitis. J Pathol 209: 540-548, 2006. 